\title{
Autoimmune markers are undetectable in end stage idiopathic dilated cardiomyopathy
}

\author{
Nicole de Leeuw, Willem J G Melchers, Dirk J Ruiter, Alida L P Caforio, \\ Aggie H M M Balk, Nicolaas de Jonge, Jochem M D Galama
}

Department of Medical Microbiology, University Hospital Nijmegen, $6500 \mathrm{HB}$ Nijmegen, Netherlands $\mathrm{N}$ de Leeuw W J G Melchers J M D Galama

Department of Pathology, University Hospital Nijmegen D J Ruiter

Department of Experimental and Clinical Medicine, Division of Cardiology, University of Padua, Padua, Italy

A L P Caforio

Department of Cardiology, University Hospital

Rotterdam-Dijkzigt,

Rotterdam,

Netherlands

A H M M Balk

Department of Cardiology, University Hospital Utrecht, Utrecht, Netherlands $\mathrm{N}$ de Jonge

Correspondence to: Dr Melchers.

email:

w.melchers@mmb.azn.nl

Accepted for publication 14 May 1999

\begin{abstract}
Background-Autoreactive humoral and cellular immune responses may be involved in the pathogenesis of idiopathic dilated cardiomyopathy (IDC). Certain human leucocyte antigens (HLA) could also be linked to the development of IDC. Aim-To determine whether various markers of autoimmunity are present in the final phase of the disease, to substantiate the role of an autoimmune process in IDC.

Methods-37 patients with end stage IDC were studied, together with 39 patients with end stage heart disease of known aetiology who were included for comparison. Multiple myocardial tissue samples from the explanted heart of each patient were evaluated (immuno)histologically. An indirect immunofluorescence assay was used to screen patient serum samples for the presence of heart specific autoantibodies. HLA class I and II frequencies were determined in each group and compared with HLA frequencies from healthy blood donors.

Results-Only scanty small mononuclear cell infiltrates were present in myocardial tissue of seven patients with IDC and of 11 patients with heart disease of known cause. The majority of these inflammatory cells were negative for $\mathbf{T}$ cell markers. All blood specimens were negative for heart specific autoantibodies and there was no apparent association of IDC with particular HLA phenotypes.

Conclusions-These findings suggest that an active autoimmune process is not involved in the end stage of IDC.

(f Clin Pathol 1999;52:739-743)
\end{abstract}

Keywords: idiopathic dilated cardiomyopathy; autoimmunity; autoantibodies

A great variety of infectious and non-infectious agents may initiate processes in the heart that eventually result in a shared clinical picture known as dilated cardiomyopathy. Dilated cardiomyopathy is the second most important cause of heart failure in the industrialised world. Microbial infection, alcoholism, drugs, toxins, genetic predisposition, and many other factors can play a role in its development. ${ }^{1}$ However, in the majority of cases the aetiology is unknown and the disease is referred to as idiopathic dilated cardiomyopathy (IDC). It is believed, however, that IDC can be a sequel of myocarditis. Viral infections are the most common cause of myocarditis. ${ }^{2}$ Apart from their direct cytolytic effect on cardiomyocytes, viruses may indirectly cause cell damage by activating cytotoxic $\mathrm{T}$ lymphocytes. ${ }^{3}$ Murine models of myocarditis have demonstrated the ability of such $\mathrm{T}$ cells not only to kill virus infected cells but also to destroy non-infected cells in normal recipient mice. ${ }^{4}$ In addition, antibodies directed against certain cardiopathogenic microorganisms were found to cross react with heart specific antigens. ${ }^{56}$ This led to the hypothesis that the cellular and humoral immune responses could themselves play a part in the development of myocardial damage during and after an infection. ${ }^{7}$ Indeed, patients with myocarditis and dilated cardiomyopathy were found to have significantly higher levels of heart specific autoantibodies than controls. ${ }^{8-10}$ In an in vitro study, antiheart autoantibodies present in the sera of patients with dilated cardiomyopathy were shown to preferentially kill a cardiac myocyte cell line. ${ }^{9}$ In another study, autoantibodies against the $\beta_{1}$ adrenoceptor, purified from sera of patients with IDC, increased the beating frequency of isolated rat heart myocytes in a concentration dependent manner. ${ }^{11}$ Therefore, virus induced activation of the immune response leading to autoimmunity may be involved in the disease process of myocarditis or IDC. ${ }^{412}$ Because certain autoimmune diseases are strongly associated with specific human leucocyte antigen (HLA) molecules, ${ }^{13}$ several research groups have looked for an HLA association with IDC. ${ }^{14-17}$ HLA-DR4 was most often found to be associated with IDC. In these studies, the DR4 antigen frequency ranged from $21 \%$ to $51 \%$ in patients with IDC, compared with $11 \%$ to $27 \%$ in controls.

We investigated heart tissue and serum samples from patients undergoing heart transplantation to determine whether autoimmune factors are present in the end stage of IDC.

\section{Methods}

PATIENTS

We included 76 white Dutch patients with end stage heart failure in this study. Thirty seven patients (21 male and 16 female; mean age 47 years (range 14 to 63)) had a diagnosis of IDC. They had no signs of obstructive coronary artery disease, congenital or primary valvar heart disease, systemic disease, insulin dependent diabetes mellitus, or alcohol abuse. The hearts of these patients had dilated, diffusely hypokinetic chambers, a left ventricular ejection fraction below $20 \%$, and a normal or decreased wall thickness. The comparison group comprised 39 patients (32 male and 


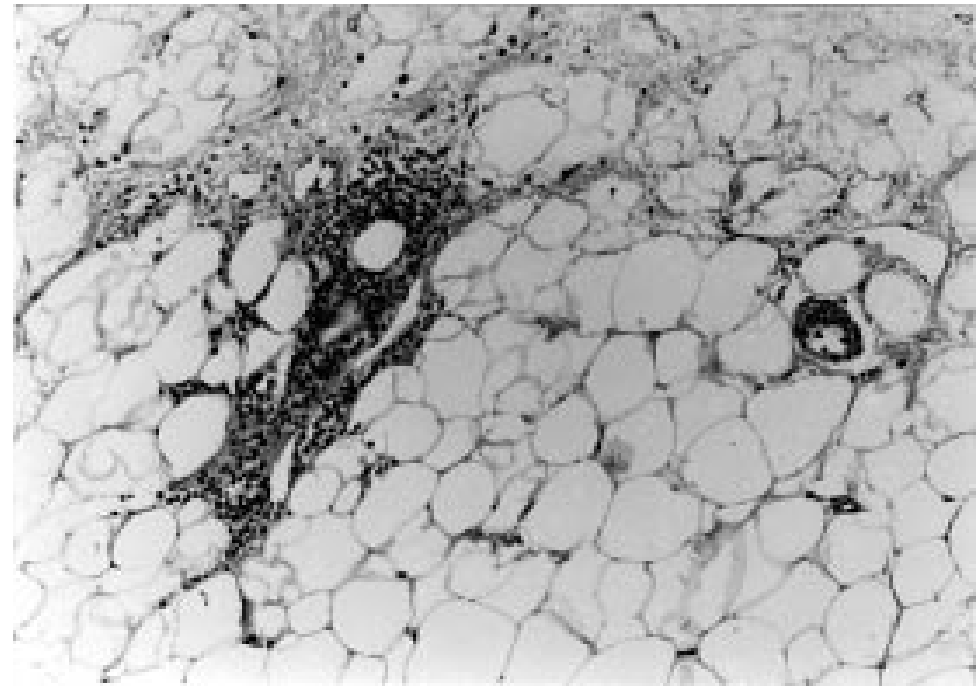

Figure 1 Mononuclear inflammatory infiltrate in the subepicardial layer of a right heart ventricle tissue sample from a patient with idiopathic dilated cardiomyopathy.

(Haematoxylin and eosin stain.)

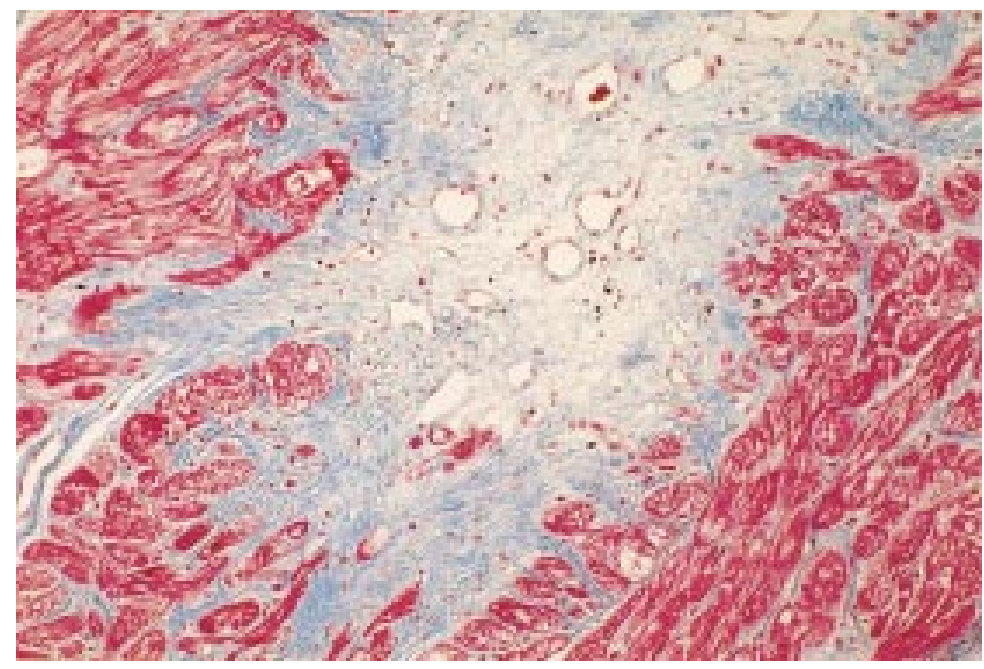

Figure 2 Paraffin section of a left heart ventricle tissue sample from a patient with ischaemic heart disease. Individually located inflammatory cells are seen in an area of reparative changes with initiation of fibrosis. Two months before heart transplantation, this patient underwent heart surgery and a left ventricular assist device was implanted. (Masson's trichrome stain.) specimens were excised from the left and right heart ventricles within one hour after explantation. The length and width of the tissue samples varied from 5 to $10 \mathrm{~mm}$; the depth equalled the wall thickness of the heart (ranging from 3 to $20 \mathrm{~mm}$ ).

\section{HISTOLOGY}

Immediately after excision, the heart tissue samples were fixed in buffered formalin and subsequently embedded in paraffin and cut into serial sections $4 \mu \mathrm{m}$ thick. From each patient, two left and two right heart ventricle tissue sections were stained with haematoxylin and eosin; likewise, four consecutive tissue sections were stained with Masson's trichrome according to standard protocols. All heart tissue sections (average size $80 \mathrm{~mm}^{2}$ ) were examined in a blinded fashion by two observers.

\section{IMMUNOHISTOCHEMISTRY}

Myocardial tissue sections which contained inflammatory cell infiltrates were further evaluated by means of immunohistochemical analysis. Antibodies against CD3 (pan T cells) and CD8 (suppressor/cytotoxic T cells) were used to identify $\mathrm{T}$ lymphocytes in the myocardium. Paraffin embedded heart tissue sections were dewaxed in xylene and rinsed in ethanol. Endogenous peroxidase activity was blocked for 30 minutes at room temperature in 3\% $\mathrm{H}_{2} \mathrm{O}_{2}$ and the slides were then rinsed in phosphate buffered saline (PBS). To improve the staining patterns, the slides were boiled in $10 \mathrm{mM}$ citrate buffer, $\mathrm{pH} 6$. After preincubation for 10 minutes in $20 \%$ normal goat serum at room temperature, the tissue sections were incubated with rabbit antihuman CD3 immunoglobulin (1:100; Dakopatts) for one hour. The slides were washed in PBS and subsequently incubated with biotinylated goat antirabbit IgG (1:200; Vector Laboratories) for 30 minutes and washed again in PBS. To demonstrate the biotinylated antibodies, the avidinbiotin-peroxidase method was applied, ${ }^{18}$ using the Elite ABC kit (Vector Laboratories). Peroxidase activity was demonstrated by diaminobenzidine substrate resulting in a brown reaction product. Finally, a haematoxylin counterstain was performed and the sections were coverslipped in Permount (Fisher Scientific). For the detection of CD8 a monoclonal mouse antihuman CD8 IgG (1:40; Dakopatts) was used in a similar way. years in the IDC group (range one month to $>10$ years) and more than 10 years in the comparison group (range three months to $>10$ years).

This study was performed after approval by the committees for scientific research with humans of the University Hospitals of Rotterdam, Utrecht, and Nijmegen. Informed consent was obtained from all patients or their parents or guardians.

CLINICAL SPECIMENS

A few hours before heart transplantation, blood samples were collected from each patient. Multiple transmural myocardial tissue
DETECTION OF HEART SPECIFIC AUTOANTIBODIES An indirect immunofluorescence assay as described by Caforio et $a l^{19}$ was used to detect heart specific autoantibodies in serum samples collected preoperatively from the heart transplant recipients. Briefly, the sera were tested at $1 / 10$ dilution on $4 \mu \mathrm{m}$ cryostat sections of blood group $\mathrm{O}$ normal human atrium and skeletal muscle. Sections were then washed with PBS and incubated with fluorescein isothiocyanate labelled sheep antihuman immunoglobulin. After subsequent washing, the sections were examined by fluorescence microscopy. The intensity of immunofluorescence of 
Table 1 HLA class I frequencies of two groups of patients with end stage heart disease, and of healthy blood donors

\begin{tabular}{|c|c|c|c|c|c|c|c|}
\hline$H L A$ & $I D C(n=37)$ & $\begin{array}{l}\text { Comparison } \\
(n=39)\end{array}$ & $\begin{array}{l}\text { Control } \\
(n=2440)\end{array}$ & $H L A$ & $I D C(n=37)$ & $\begin{array}{l}\text { Comparison } \\
(n=39)\end{array}$ & $\begin{array}{l}\text { Control } \\
(n=2440)\end{array}$ \\
\hline $\mathrm{A} 1$ & $13(35 \%)$ & $14(36 \%)$ & $756(31 \%)$ & B5 & $5(14 \%)$ & $6(15 \%)$ & $293(12 \%)$ \\
\hline A2 & $20(54 \%)$ & $16(41 \%)$ & $1293(53 \%)$ & B7 & $11(30 \%)$ & $7(18 \%)$ & $659(27 \%)$ \\
\hline A3 & $9(24 \%)$ & $9(23 \%)$ & $707(29 \%)$ & B8 & $11(30 \%)$ & $14(36 \%)$ & $561(23 \%)$ \\
\hline A9 & $7(19 \%)$ & $10(26 \%)$ & $464(19 \%)$ & B12 & $9(24 \%)$ & $12(31 \%)$ & $610(25 \%)$ \\
\hline A10 & $3(8 \%)$ & $3(8 \%)$ & $171(7 \%)$ & B13 & $1(3 \%)$ & $2(5 \%)$ & $122(5 \%)$ \\
\hline A11 & $5(14 \%)$ & $6(15 \%)$ & $293(12 \%)$ & B14 & $1(3 \%)$ & 0 & $73(3 \%)$ \\
\hline A19 & $7(19 \%)$ & $9(23 \%)$ & $512(21 \%)$ & B15 & $5(14 \%)$ & $6(15 \%)$ & $390(16 \%)$ \\
\hline \multirow[t]{2}{*}{ A 28} & $4(11 \%)$ & $2(5 \%)$ & $244(10 \%)$ & B16 & $4(11 \%)$ & $3(8 \%)$ & $171(7 \%)$ \\
\hline & & & & B17 & $1(3 \%)$ & $2(5 \%)$ & $171(7 \%)$ \\
\hline Cw1 & $3(8 \%)$ & $2(5 \%)$ & $122(5 \%)$ & B18 & $1(3 \%)$ & $6(15 \%)$ & $171(7 \%)$ \\
\hline $\mathrm{Cw} 2$ & $4(11 \%)$ & $4(10 \%)$ & $244(10 \%)$ & B21 & $3(8 \%)$ & 0 & $49(2 \%)$ \\
\hline Cw3 & $11(30 \%)$ & $10(26 \%)$ & $854(35 \%)$ & $\mathrm{B} 22$ & $2(5 \%)$ & $1(3 \%)$ & $146(6 \%)$ \\
\hline $\mathrm{Cw} 4$ & $7(19 \%)$ & $12(31 \%)$ & $537(22 \%)$ & B27 & $4(11 \%)$ & 0 & $146(6 \%)$ \\
\hline Cw5 & $6(16 \%)$ & $4(10 \%)$ & $342(14 \%)$ & B35 & $6(16 \%)$ & $9(23 \%)$ & $439(18 \%)$ \\
\hline Cw6 & $2(5 \%)$ & $7(18 \%)$ & $415(17 \%)$ & B37 & 0 & $1(3 \%)$ & $98(4 \%)$ \\
\hline $\mathrm{Cw} 7$ & $23(62 \%)$ & $22(56 \%)$ & $1244(51 \%)$ & B40 & $4(11 \%)$ & $7(18 \%)$ & $439(18 \%)$ \\
\hline Cw8 & 0 & 0 & $24(1 \%)$ & B41 & 0 & $2(5 \%)$ & $24(1 \%)$ \\
\hline
\end{tabular}

HLA, human leucocyte antigen; IDC, idiopathic dilated cardiomyopathy.

the positive standard at 1/40 dilution was used as the threshold for positivity.

\section{HLA TYPING}

From each patient undergoing cardiac transplantation, HLA class I and II phenotypes were determined by conventional serological methods, based on complement dependent cytotoxicity. The frequencies of HLA phenotypes of 2440 random, healthy, unrelated, white Dutch blood donors, reported by Schipper et al, ${ }^{20}$ were used as a control dataset.

STATISTICS

The difference in frequency of the various HLA antigens between two groups was tested for significance by means of the two tailed $\chi^{2}$ test at the $5 \%$ level. Probability (p) values were corrected for the number of comparisons made.

\section{Results}

HISTOLOGY

In each patient, histological evaluation of the paraffin embedded heart tissue sections revealed a varying degree of myocyte hypertrophy and significant fibrosis in at least one of the four myocardial tissue samples. In the majority of heart tissue sections, inflammatory cell infiltrates were absent. Single or few small infiltrates (less than 100 mononuclear cells) were found in some of the tissue sections from

Table 2 HLA class II frequencies of two groups of patients with end stage heart disease, and of healthy blood donors

\begin{tabular}{lccc}
\hline HLA & $\begin{array}{l}\text { IDC } \\
(n=37)\end{array}$ & $\begin{array}{l}\text { Comparison } \\
(n=39)\end{array}$ & $\begin{array}{l}\text { Control } \\
(n=2440)\end{array}$ \\
\hline DR1 & $7(19 \%)$ & $5(13 \%)$ & $488(20 \%)$ \\
DR2 & $16(43 \%)$ & $7(18 \%)$ & $708(29 \%)$ \\
DR3 & $11(30 \%)$ & $14(36 \%)$ & $610(25 \%)$ \\
DR4 & $7(19 \%)$ & $10(26 \%)$ & $683(28 \%)$ \\
DR5 & $7(19 \%)$ & $7(18 \%)$ & $464(19 \%)$ \\
DR6 & $10(27 \%)$ & $12(31 \%)$ & $830(34 \%)$ \\
DR7 & $6(16 \%)$ & $11(28 \%)$ & $464(19 \%)$ \\
DR8 & $1(3 \%)$ & $4(10 \%)$ & $122(5 \%)$ \\
DR9 & $3(8 \%)$ & $1(3 \%)$ & $49(2 \%)$ \\
DR10 & $1(3 \%)$ & $1(3 \%)$ & $98(4 \%)$ \\
& & & \\
DQ1 & $27(73 \%)$ & $22(56 \%)$ & $1732(71 \%)$ \\
DQ2 & $13(35 \%)$ & $20(51 \%)$ & $903(37 \%)$ \\
DQ3 & $15(41 \%)$ & $21(54 \%)$ & $1244(51 \%)$ \\
DQ4 & 0 & $1(3 \%)$ & $73(3 \%)$ \\
\hline
\end{tabular}

HLA, human leucocyte antigen; IDC, idiopathic dilated cardiomyopathy.
18 patients (seven from the IDC group and 11 from the comparison group). The infiltrates were mostly located in the (sub)epicardial layer near blood vessels or in areas of reparative changes with onset of fibrosis (figs 1 and 2). The infiltrates consisted of lymphocytes, polymorphonuclear leucocytes, macrophages, and mast cells. Additional immunohistochemical analysis showed that $T$ cells were scarcely represented in these infiltrates. In none of these patients was the mean number of $\mathrm{T}$ lymphocytes greater than $5.0 / \mathrm{mm}^{2}$ of myocardial tissue.

HEART SPECIFIC AUTOANTIBODIES

No heart specific or cross reactive autoantibodies were detected in any of the serum samples from the patients with end stage heart failure.

HLA TYPING

The broad antigen frequencies of HLA class I and class II molecules are summarised in tables 1 and 2, respectively. No significant difference was observed between the IDC group, the comparison group, or the control dataset.

\section{Discussion}

We did not find markers of autoimmunity in our study group of 37 patients with end stage IDC. In particular, heart specific autoantibodies were absent. A cellular autoimmune process seemed unlikely because $\mathrm{T}$ cells were scarcely present or were absent in the few small mononuclear cell infiltrates that were found in myocardial tissue of seven patients with IDC. Furthermore, there was no significant association of IDC with particular HLA phenotypes in our study population. The absence of autoimmune markers in this study could have been a chance finding because the group with IDC comprised a relatively small number of patients. However, there are other possible explanations for our findings.

It might be that autoimmunity gradually subsides and that autoantibodies as well as cellular infiltrates disappear by the end stage of the disease. Indeed, Caforio et $a l^{21}$ recently reported that heart specific autoantibodies in dilated cardiomyopathy become undetectable with disease progression, a feature also characteristic of established autoimmune disorders 
such as insulin dependent diabetes mellitus. ${ }^{13}$ Although the absence of autoantibodies in our patients with end stage heart disease agrees with the findings of Caforio et al, ${ }^{21}$ eight of our IDC patients reached the end stage within one year of the onset of symptoms. If an autoimmune response is responsible for myocardial injury in IDC, one would expect to find it in those patients with a relatively short clinical course of IDC. However, the actual onset of the disease may precede the clinical symptoms by years. In addition, cardiac antibodies are more prevalent in the familial dilated cardiomyopathy $^{8}$ and it appeared that none of the eight IDC patients had the familial form of the disease. Whether these antibodies are cause or consequence of myocardial injury is still uncertain. ${ }^{22}$ In vitro experiments have shown that various autoantibodies can penetrate living cells. ${ }^{23}$ After they bind to their intracellular antigenic targets, cell functions may be modified and this can ultimately cause cell death. Perhaps autoantibodies against cardiac myosin ${ }^{524}$ act in such a way. It is also possible that some autoantibodies specific for intracellular antigens are secondary to myocardial disease without being pathogenic. Other antibodies that interact with extracellular antigens may cause disease by acting as receptor agonists. ${ }^{11}$ Functional characterisation of heart specific autoantibodies is essential in determining their true role in cardiac disease.

Gradual subsidence of autoimmunity may also account for the fact that there is little evidence of cellular autoimmunity in IDC. Activated $\mathrm{T}$ cells have been shown to be present in myocardial tissue of patients with dilated cardiomyopathy. ${ }^{25}{ }^{26}$ In our study we found that there were very few lymphocytes in the explanted hearts of our patients. In addition, immunohistochemical analysis of the minor mononuclear cell infiltrates in eight of these patients showed that most of these cells were negative for the $\mathrm{T}$ lymphocyte markers CD3 and CD8. In only one patient with ischaemic heart disease were numerous CD3 and CD 8 positive cells detected among many macrophages, predominantly in the left ventricular tissue sections. Two months before heart transplantation, this patient underwent heart surgery and a left ventricular assist device was implanted because of severe malfunction of the left ventricle.

Unlike humoral and cellular immune effectors that might disappear in the course of a disease, HLA phenotypes are stable characteristics of an individual. Several research groups have sought an HLA association with IDC and in most studies an increased frequency of HLA-DR4 was found in patients with IDC. ${ }^{14} 1517$ Various other HLA phenotypes were also reported to be associated with the disease, but these differed from group to group. ${ }^{14-16}$ The lack of consistency among these results may reflect the different ethnic origins of the study populations as well as the heterogeneity in aetiology and pathogenesis of IDC. However, a strong and convincing HLA link, as is found in diseases like ankylosing spondylitis, coeliac disease, and insulin dependent diabetes mellitus, is missing. ${ }^{13}$ We did not find any significant relation between a particular HLA phenotype and IDC. Our data are in agreement with results reported by $\mathrm{McK}-$ enna et al. ${ }^{17}$ An HLA relation with a subpopulation of cases of IDC - for example, the familial form of the disease-may be inapparent owing to heterogeneity within the entire IDC population, as was shown by McKenna et al. ${ }^{17}$ They showed that the overall frequency of HLA-DR4 in patients with IDC was similar to that in controls. However, the HLA type DR4 was significantly more common in patients with familial dilated cardiomyopathy than in patients with the non-familial form. Although we did not perform family screening, limited information was available from the clinical records on cardiovascular disease in family members, and based on these data our IDC patients did not appear to have the familial form of the disease. Instead of individual HLA phenotypes, specific combinations of HLA phenotypes could produce increased susceptibility to IDC. In addition, non-MHC genes are likely to be involved in the development or maintenance of IDC. ${ }^{412}$ The importance of genetic factors in IDC has long been underestimated, but data are accumulating that confirm the role of these factors in a growing subset of the IDC population. ${ }^{27} 28$

In conclusion, despite suggestive evidence that the immune system is involved in the early phase of a disease process that leads to IDC, we did not detect markers of an active autoimmune reaction in patients who clinically were in the end stage of the disease. Autoimmune diseases are often linked to certain genetic traits. Therefore, identification of hereditary IDC $^{29}$ and subsequent family studies, as well as molecular genetic analyses, can provide us with opportunities to examine the early phase of IDC and determine to what extent the immune system contributes to the development of the disease.

This study was supported by a grant from the Netherlands Heart Foundation (92-301). We thank Professor William McKenna for giving us the opportunity to perform the indirect immunofluorescence assay in his laboratory. Special thanks to
Aldwyn J Haven for his help in performing the IFAs and to Coos Diepenbroek and Cathy Maass for histotechnical support.

1 Kasper EK, Agema WRP, Hutchins GM, et al. The causes of dilated cardiomyopathy: a clinicopathologic review of 673 consecutive patients. F Am Coll Cardiol 1994;23:586-90.

2 Savoia MC, Oxman MN. Myocarditis and pericarditis. In: Mandell GL, Bennet JE, Dolin R, eds. Principles and practice of infectious diseases. New York: Churchill Livingstone, 1995:799-813.

3 Woodruff, JF. Viral myocarditis. A review. Am f Pathol 1980; 101:425-84.

4 Huber S. Animal models of human disease. Autoimmunity in myocarditis: relevance of animal models. Clin Immunol in myocarditis: relevance of anim

5 Neumann DA, Rose NR, Ansari AA, et al. Induction of multiple heart autoantibodies in mice with coxsackievirus B3- and cardiac myosin-induced autoimmune myocarditis. f Immunol 1994;152:343-50.

6 Ferrari I, Levin MJ, Wallukat G, et al. Molecular mimicry between the immunodominant ribosomal protein $\mathrm{P} 0$ of Trypanosoma cruzi and a functional epitope on the human $\beta_{1}$-adrenergic receptor. F Exp Med 1995;182:59-65.

7 Rose NR, Neumann DA, Herskowitz A. Autoimmune myocarditis: concepts and questions. Immunol Today 1991; 12:253-5.

8 Caforio ALP, Keeling PJ, Zachara E, et al. Evidence from family studies for autoimmunity in dilated cardiomyopathy. Lancet 1994;344:773-7. 
9 Latif N, Smith J, Dunn J, et al. Complement-mediated cytotoxic activity of anti-heart antibodies present in the sera of patients with $19 \cdot 99-104$

10 Pankuweit S, Portig I, Lottspeich F, et al. Autoantibodies in sera of patients with myocarditis: Characterization of the corresponding proteins by isoelectric focusing and
$\mathrm{N}$-terminal sequence analysis. $\mathcal{F} \mathrm{Mol}$ Cell Cardiol 1997;29: N-term

11 Magnusson Y, Wallukat G, Waagstein F, et al. Autoimmunity in idiopathic dilated cardiomyopathy: characterization of antibodies against the $\beta$-adrenoceptor with positive chronotropic effect. Circulation 1994;89:2760-7.

12 Cetta F, Michels VV. The autoimmune basis of dilated cardiomyopathy. Ann Med 1995;27:169-73.

13 Thorsby E, Undlien D. The HLA associated predisposition to type 1 diabetes and other autoimmune diseases. $\mathcal{F}$ Pediatr Endocrinol Metab 1996;9:75-88.

14 Carlquist JF, Menlove RL, Murray MB, et al. HLA class II (DR and DQ) antigen associations in idiopathic dilated cardiomyopathy. Validation study and meta-analysis of pubdiomyopathy. Validation study and meta-analysis of pub-
lished HLA association studies. Circulation 1991;83:515-22.

15 Caforio ALP, Martinetti M, Schwarz G, et al. Idiopathic dilated cardiomyopathy: lack of association between circulating organ-specific cardiac antibodies and HLA-DR antigens. Tissue Antigens 1992;39:236-40.

16 Carlquist JF, Ward RH, Husebye D, et al. Major histocompatibility complex class II gene frequencies by serologic and deoxyribonucleic acid genomic typing in idiopathic dilated cardiomyopathy. Am $\mathcal{F}$ Cardiol 1994;74:918-20.

17 McKenna CJ, Codd MB, McCann HA, et al. Idiopathic dilated cardiomyopathy: familial prevalence and HLA distribution. Heart 1997;77:549-52.

18 Hsu SM, Raine L, Fanger H. Use of avidin-biotin-peroxidase complex $(\mathrm{ABC})$ in immunoperoxidase techniques: a comparison between $\mathrm{ABC}$ and unlabeled antibody (PAP) procedures. F Histochem Cytochem 1981;29:577-80.
19 Caforio ALP, Bonifacio E, Stewart JT, et al. Novel organ-specific circulating cardiac autoantibodies in dilated cardiomyopathy. $\mathcal{F}$ Am Coll Cardiol 1990;15:1527-34.

20 Schipper RF, Schreuder GMT, D'Amaro J, et al. HLA gene and haplotype frequencies in Dutch blood donors. Tissue Antigens 1996;48:562-74.

21 Caforio ALP, Goldman JH, Baig MK, et al. Cardiac autoantibodies in dilated cardiomyopathy become undetectable with disease progression. Heart 1997;77:62-7.

22 Limas CJ. Cardiac autoantibodies in dilated cardiomyopathy. A pathogenic role? Circulation 1997;95:1979-80.

23 Alarcón-Segovia D, Ruiz-Argüelles A, Llorente L. Broken dogma: penetration of autoantibodies into living cells. Immunol Today 1996;17:163-4.

24 Caforio ALP, Grazzini M, Mann JM, et al. Identification of $\alpha$ - and $\beta$-cardiac myosin heavy chain isoforms as major autoantigens in dilated cardiomyopathy. Circulation 1992; 85:1734-42.

25 Holzinger C, Schöllhammer A, Imhof M, et al. Phenotypic patterns of mononuclear cells in dilated cardiomyopathy. Circulation 1995;92:2876-85.

26 Badorff C, Noutsias M, Kühl U, et al. Cell-mediated cytotoxicity in hearts with dilated cardiomyopathy: correlation with interstitial fibrosis and foci of activated $\mathrm{T}$ lymphocytes. F Am Coll Cardiol 1997;29:429-34.

27 Olson TM, Michels VV, Thibodeau SN, et al. Actin mutations in dilated cardiomyopathy, a heritable form of heart failure. Science 1998;280:50-2.

28 Arbustini E, Diegoli M, Fasani R, et al. Mitochondrial DNA mutations and mitochondrial abnormalities in dilated cardiomyopathy. Am f Pathol 1998;153:501-10

29 Mestroni L, Maisch B, McKenna WJ, et al. Guidelines for the study of familial dilated cardiomyopathies. Eur Heart $\mathcal{F}$ 1999;20:93-102. 